\title{
Medullary thyroid cancer: an introduction
}

\author{
M.K. Gupta MD MSc*
}

Medullary thyroid cancer (MTC) is a rare cancer, making up perhaps only a few percent of all thyroid cancers ${ }^{1}$. Despite low incidence rates for MTC, physicians treating thyroid cancer must be both knowledgeable and accomplished in the treatment of this disease.

Arising from the calcitonin-producing $\mathrm{C}$ cells of the thyroid, MTc behaves quite differently from the more common types of thyroid cancer. Strategies designed for well differentiated cancer will not be successful for MTc. Thus, it is crucial for physicians to understand that MTC represents a significant departure from the familiar paradigm of well-differentiated thyroid cancer. In 2015, the American Thyroid Association released consensus guidelines to help direct the management of MTC $^{1}$. The guidelines are easy to read and thoughtful, and they take a balanced approach to MTC management.

Thomas et al. ${ }^{2}$ and Larouche et al. ${ }^{3}$ have both done an excellent job of building on the American Thyroid Association document. Thomas et al. have concentrated on the clinical aspects of MTC management, and Larouche et al. have focused on the physiology of the RET proto-oncogene.

Key points from the paper by Thomas et al. (this issue of Current Oncology) include noting the weaknesses of fine-needle aspiration techniques in diagnosing MTC and the primacy of biochemical markers in treatment and surveillance. Larouche et al. (next issue of Current Oncology) provide an outstanding review of the genetic basis of the disease and a review of the management strategies for each codon mutation.

Together, both papers are an excellent addition to the current literature and an easily digestible refresher in the management of an unusual, but interesting disease.

\section{CONFLICT OF INTEREST DISCLOSURES}

I have read and understood Current Oncology's policy on disclosing conflicts of interest, and I declare that I have none.

\section{AUTHOR AFFILIATIONS}

*Department of Surgery, Division of Otolaryngology, McMaster University, Hamilton, ON.

\section{REFERENCES}

1. Wells SA Jr, Asa SL, Dralle H, et al. Revised American Thyroid Association guidelines for the management of medullary thyroid carcinoma. Thyroid 2015;25:567-610.

2. Thomas CM, Asa SL, Ezzat S, Sawka AM, Goldstein D. Diagnosis and pathologic characteristics of medullary thyroid carcinoma-review of current guidelines. Curr Oncol 2019;26:338.

3. Larouche V, Akirov A, Thomas CM, Krzyzanowska MK, Ezzat S. A primer on the genetics of medullary thyroid cancer. Curr Oncol 2019;26:[in press]. 